\title{
Regadenosón intravenoso frente a adenosina intracoronaria para la medida de la reserva fraccional de flujo
}

\author{
Pau Federico Zaragoza*, Luis Martínez Ortiz de Urbina, Teresa Castelló Viguer, \\ Francisco Pomar Domingo y Enrique Peris Domingo
}

Servicio de Cardiología, Hospital Universitario La Ribera, Alzira, Valencia, España

\section{RESUMEN}

Introducción y objetivos: El regadenosón, un agonista selectivo de los receptores A2a de la adenosina, se ha propuesto como una alternativa para el cálculo de la reserva fraccional de flujo (RFF). El objetivo de este estudio fue evaluar la utilidad del regadenosón en comparación con el uso de adenosina intracoronaria.

Métodos: Se incluyeron de forma prospectiva y consecutiva 41 lesiones coronarias intermedias (30-70\%) en las que se indicó valoración funcional con guía de presión. A cada paciente se le administró de forma secuencial adenosina intracoronaria y regadenosón intravenoso, y se registraron los datos hemodinámicos y los efectos adversos con la hiperemia inducida mediante ambos fármacos. Las diferencias en la RFF final se analizaron mediante regresión lineal y se identificaron las discrepancias clínicamente relevantes asumiendo como punto de corte el valor de 0,80 .

Resultados: La media de la RFF fue significativamente menor con regadenosón que con adenosina $(0,838 \pm 0,072$ frente a 0,852 $\pm 0,073$; $\mathrm{p}=0,002)$, y en 4 casos $(9,8 \%)$ se encontraron diferencias clínicamente relevantes. El análisis de regresión mostró una fuerte correlación lineal entre los valores individuales $(r=0,925 ; \mathrm{p}<0,001)$. Tanto la adenosina como el regadenosón redujeron de forma significativa la presión arterial media, y solo el regadenosón incrementó significativamente la frecuencia cardiaca basal. En 2 casos $(4,9 \%)$ se registró asistolia > $3 \mathrm{~s}$ tras recibir adenosina y no se observó ninguna complicación con regadenosón.

Conclusiones: La administración de regadenosón en bolo único intravenoso mostró una significativa reducción en el valor de RFF en comparación con la administración de bolos de adenosina intracoronaria, y las diferencias observadas podrían resultar relevantes en la toma de decisiones clínicas.

Palabras clave: Adenosina. Regadenosón. Reserva fraccional de flujo.

\section{Intravenous regadenoson versus intracoronary adenosine for fractional flow reserve measurement}

\begin{abstract}
Introduction and objectives: Regadenoson, a selective agonist of the A2a receptors of adenosine, has been proposed as an alternative for the measurement of fractional flow reserve (FFR). The goal of our study was to assess the utility of regadenoson compared to the use of intracoronary adenosine.

Methods: Forty-one intermediate coronary lesions (30\%-70\%), in which functional assessment with pressure wire was indicated, were included both prospective and consecutively. Each patient was sequentially administered intracoronary adenosine and intravenous regadenoson and hemodynamic data while the adverse effects were recorded with hyperemia induced by both drugs. The differences seen in the final FFR were analyzed using the linear regression model and the clinically relevant discrepancies were identified assuming 0.80 as the cut-off point.

Results: The mean of the FFR was significantly lower with regadenoson compared to adenosine $(0.838 \pm 0.072$ vs $0.852 \pm 0.073$ $P=.002)$ and in 4 cases $(9.8 \%)$ clinically relevant differences were found. The regression analysis showed a strong linear correlation between the individual values $(\mathrm{r}=0.925, P<.001)$. Both adenosine and regadenoson significantly reduced mean arterial blood pressure and only regadenoson significantly increased baseline heart rate. In 2 cases (4.9\%) asystole was recorded $>3$ seconds after the administration of adenosine and no complications were observed with regadenoson.

Conclusions: The administration of regadenoson through an intravenous single bolus has shown a significant reduction in the value of FFR compared to the administration of intracoronary adenosine boluses and the observed differences may be relevant in the clinical decision-making process.
\end{abstract}

Keywords: Adenosine. Regadenoson. Fractional flow reserve.

Abreviaturas

RFF: reserva fraccional de flujo.

* Autor para correspondencia: Servicio Cardiología, Hospital Universitario La Ribera, Ctra. Corbera km. 1, 46600 Alzira, Valencia, España.

Correo electrónico: pau@comv.es (P. Federico Zaragoza).

Recibido el 14 de enero de 2019. Aceptado el 11 de febrero de 2019. Online: 21-05-2019.

Full English text available from: www.recintervcardiol.org/en.

https://doi.org/10.24875/RECIC.M19000021

2604-7306 / @ 2019 Sociedad Española de Cardiología. Publicado por Permanyer Publications. Este es un artículo open access bajo la licencia CC BY-NC-ND 4.0. 


\section{INTRODUCCIÓN}

La medida de la reserva funcional de flujo (RFF) se ha consolidado como una valiosa herramienta para la valoración funcional de las estenosis coronarias intermedias ${ }^{1}$. Para su cálculo se necesita una hiperemia máxima. El agente farmacológico más utilizado para inducir vasodilatación es la adenosina en infusión intravenosa o en inyección intracoronaria ${ }^{2}$.

El regadenosón, un agonista selectivo de los receptores A2a de la adenosina, se ha propuesto como una alternativa con la ventaja de su facilidad de uso, pues se precisa un único bolo intravenoso por vía periférica a dosis fija independientemente del peso y de la función renal del paciente ${ }^{3-7}$.

Varios estudios han comparado el regadenosón y la adenosina intravenosa ${ }^{8}$, pero a los autores de este artículo no les consta ningún trabajo que haya comparado el regadenosón con adenosina intracoronaria en la valoración de la RFF.

El objetivo principal consistió en establecer la variabilidad individual en la medida de la RFF mediante adenosina intracoronaria y regadenosón intravenoso administrados de forma secuencial, de manera que cada paciente actuara como caso y como control.

Los objetivos secundarios incluyeron el análisis de la respuesta hemodinámica, la medida de los tiempos de hiperemia y la valoración de los efectos adversos.

\section{MÉTODOS}

Se estudiaron de forma prospectiva y consecutiva 41 lesiones coronarias intermedias en 39 pacientes remitidos para coronariografía a los que se indicó valoración funcional con guía de presión. Se definió como lesión intermedia la estenosis del 30-70\% estimada visualmente o por cuantificación automática durante la angiografía. Se asumió el punto de corte establecido de $\mathrm{RFF} \leq 0,80$ para indicar revascularización. Se obtuvo el consentimiento informado de todos los pacientes incluidos en el estudio.

\section{Procedimiento}

La coronariografía se realizó de acuerdo con la práctica habitual. Se procedió a la medida de la RFF utilizando la guía Pressure Wire (St Jude Medical, St Paul, Minnesota, Estados Unidos), previa administración de heparina no fraccionada (50 UI/kg) y una vez situado el sensor distal a la lesión, y siguiendo los estándares recomendados para la adquisición, el registro y la interpretación de los trazados de presión ${ }^{9}$.

\section{Protocolo farmacológico}

De acuerdo con estudios previos de dosis-respuesta, se estableció como apropiada una dosis inicial de $100 \mu \mathrm{g}$ de adenosina intracoronaria para la coronaria derecha y de $200 \mu \mathrm{g}$ para la coronaria izquierda ${ }^{10}$.

Con el fin de alcanzar un grado de hiperemia óptimo, los pacientes con medidas en el límite del punto de corte $(\mathrm{RFF}<0,85)$ podían recibir, a criterio del operador, dosis repetidas con incrementos de $60 \mu \mathrm{g}$ en cada bolo. Se seleccionó como RFF verdadera el valor mínimo obtenido.

Tras la administración de la adenosina se iniciaba el registro y se consideraba finalizada la fase de hiperemia cuando la RFF volvía a valores basales. A continuación, se inyectaba por vía venosa periférica un bolo de $400 \mu \mathrm{g}$ de regadenosón y se repetían las medidas.

Se obtuvo el valor de RFF mediante análisis latido a latido, y en caso de duda o artefactos se revisaron los trazados almacenados en la consola.

Adicionalmente, se registraron los valores de frecuencia cardiaca y de presión arterial, tanto basales como en la fase de hiperemia, y se monitorizaron los posibles efectos secundarios.

Por último, se recogió para su posterior análisis el tiempo requerido con cada fármaco para alcanzar la hiperemia y la duración de esta.

\section{Análisis estadístico}

Las variables continuas se presentan como media \pm desviación estándar, y las categóricas, como valor absoluto o porcentaje. La prueba t de Student para los datos emparejados se usó para comparar los valores de RFF, los cambios hemodinámicos (presión arterial y frecuencia cardiaca) y los tiempos de hiperemia observados tras la administración de adenosina y de regadenosón. Los síntomas se evaluaron con la prueba de $\chi^{2}$. Mediante regresión lineal, cálculo del coeficiente de Pearson y gráfico de Bland-Altman se analizó la correlación entre los valores de RFF con ambos fármacos. El análisis estadístico se efectuó con el programa SPSS v20 (IBM, Armonk, Nueva York, Estados Unidos), y los resultados se consideraron significativos con valores de $\mathrm{p}<0,05$.

\section{RESULTADOS}

\section{Pacientes en estudio}

En la tabla 1 se describen las características basales de los pacientes. En total se evaluaron 41 lesiones intermedias (estenosis media: $52 \pm 9 \%$ ), 29 de ellas localizadas en la descendente anterior, 7 en la coronaria derecha y 5 en la circunfleja. La dosis media de adenosina intracoronaria administrada fue de $236 \pm 60 \mu \mathrm{g}$. No hubo complicaciones relacionadas con el procedimiento.

\section{Medida de la RFF con adenosina intracoronaria frente a regadenosón intravenoso}

Trece lesiones (33\%) mostraron, con 1 o con los 2 fármacos, valores de $\mathrm{RFF} \leq 0,80$, propios de estenosis funcionalmente significativas, y se observó una fuerte correlación lineal con ambos estímulos hiperémicos $(r=0,925 ; \mathrm{p}<0,001)$ (figura 1 y figura 2). Sin embargo, la RFF medida tras la administración de regadenosón fue menor que la obtenida tras la administración de adenosina intracoronaria $(0,838 \pm 0,072$ frente a $0,852 \pm 0,073 ; p=0,002)$ (tabla 2). Además, en cuatro casos $(9,8 \%)$ se observaron discrepancias relevantes al obtener una RFF $>0,80$ con adenosina $\mathrm{y} \leq 0,80$ con regadenosón, lo que implicó la reclasificación de la lesión (tabla 3).

\section{Parámetros hemodinámicos}

Con ambos fármacos se observó un descenso significativo en la presión arterial media basal, pero fue más pronunciado con regadenosón. Sin embargo, solo el regadenosón incrementó de forma significativa la frecuencia cardiaca basal (tabla 2).

El tiempo medio hasta alcanzar la hiperemia máxima fue significativamente menor con adenosina $(15 \pm 6$ frente a $61 \pm 49 \mathrm{~s}$ $\mathrm{p}<0,001)$, y el efecto fue más prolongado con regadenosón $(44 \pm 29$ frente a $174 \pm 72 \mathrm{~s} ; \mathrm{p}<0,001)$ (figura 3). 
Tabla 1. Características de la población en estudio

\begin{tabular}{|c|c|}
\hline \multicolumn{2}{|l|}{ Muestra $(n=41)$} \\
\hline Edad (años) & $65 \pm 14$ \\
\hline Mujeres & $27 \%$ \\
\hline Índice de masa corporal $\left(\mathrm{kg} / \mathrm{m}^{2}\right)$ & $30 \pm 3$ \\
\hline \multicolumn{2}{|l|}{ Antecedentes médicos } \\
\hline Hipertensión & $73 \%$ \\
\hline Dislipemia & $58 \%$ \\
\hline Diabetes mellitus & $24 \%$ \\
\hline Tabaquismo & $36 \%$ \\
\hline Infarto previo & $33 \%$ \\
\hline Revascularización previa & $49 \%$ \\
\hline Insuficiencia cardiaca & $3 \%$ \\
\hline \multicolumn{2}{|l|}{ Vasos enfermos } \\
\hline 1 & $59 \%$ \\
\hline 2 & $25 \%$ \\
\hline 3 & $16 \%$ \\
\hline \multicolumn{2}{|l|}{ Arteria evaluada } \\
\hline Descendente anterior & $67 \%$ \\
\hline Circunfleja & $15 \%$ \\
\hline Coronaria derecha & $18 \%$ \\
\hline \multicolumn{2}{|l|}{ Grado de estenosis } \\
\hline $30-50 \%$ & $42 \%$ \\
\hline $50-70 \%$ & $58 \%$ \\
\hline $70-90 \%$ & $0 \%$ \\
\hline Diámetro del vaso (mm) & $3,3 \pm 0,5$ \\
\hline \multicolumn{2}{|c|}{ Dosis de adenosina intracoronaria ( $\mu \mathrm{g})$} \\
\hline Coronaria izquierda & $243 \pm 55$ \\
\hline Coronaria derecha & $206 \pm 76$ \\
\hline
\end{tabular}

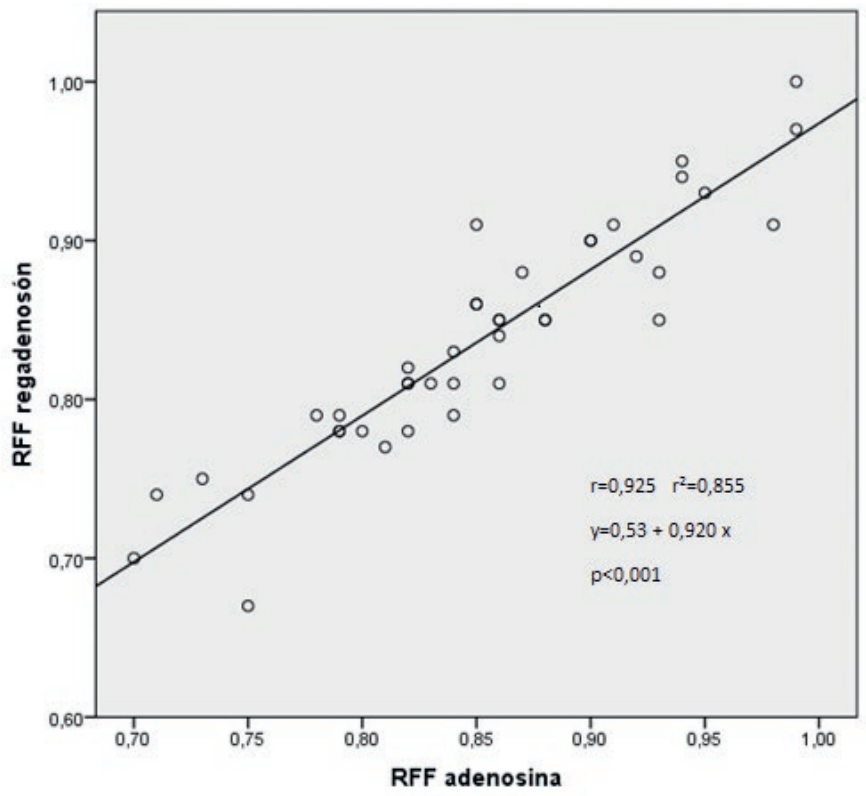

Figura 1. Análisis de regresión lineal. Correlación entre los valores de reserva fraccional de flujo medidos con adenosina intracoronaria y con regadenosón intravenoso en cada paciente. RFF: reserva fraccional de flujo.

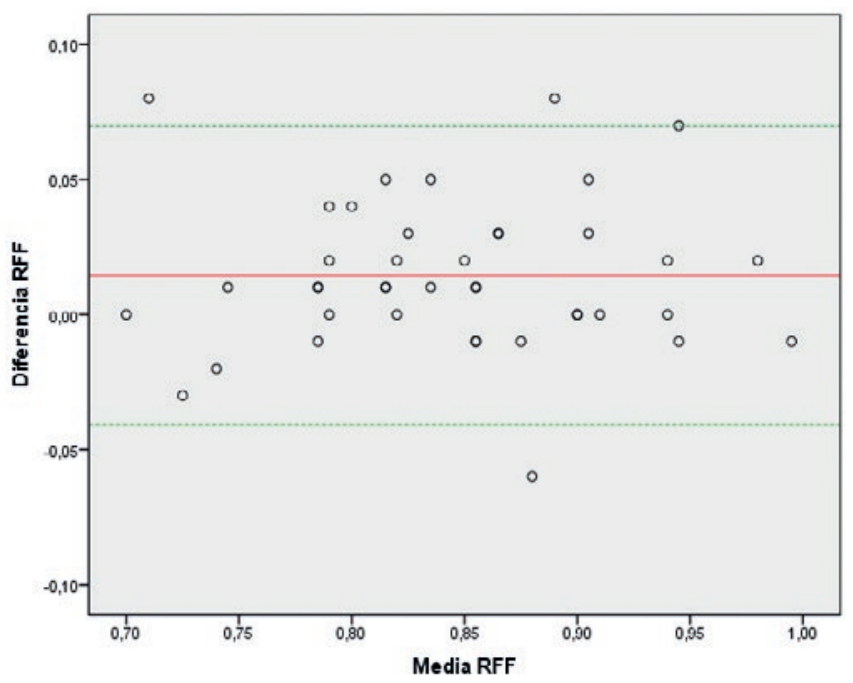

Figura 2. Análisis de Bland-Altman. Representación gráfica de las diferencias en la reserva fraccional de flujo medida con adenosina intracoronaria y con regadenosón intravenoso. RFF: reserva fraccional de flujo.

Tabla 2. Efectos hemodinámicos de la adenosina y del regadenosón

\begin{tabular}{llll} 
& Reserva fraccional de flujo & Presión arterial media $(\mathbf{m m H g})$ & Frecuencia cardiaca (latidos/min) \\
\hline Basal & $0,94 \pm 0,05$ & $114 \pm 22$ & $69 \pm 12$ \\
\hline Adenosina & $0,85 \pm 0,07$ & $92 \pm 21$ & $70 \pm 14$ \\
\hline Regadenosón & $0,84 \pm 0,07^{*}$ & $85 \pm 18^{*}$ & $89 \pm 18^{*}$ \\
\hline
\end{tabular}

Los datos expresan media \pm desviación estándar.

${ }^{*} \mathrm{p}<0,05$ respecto a basal y adenosina. 
Tabla 3. Valores individuales de reserva fraccional de flujo con adenosina intracoronaria y regadenosón intravenoso

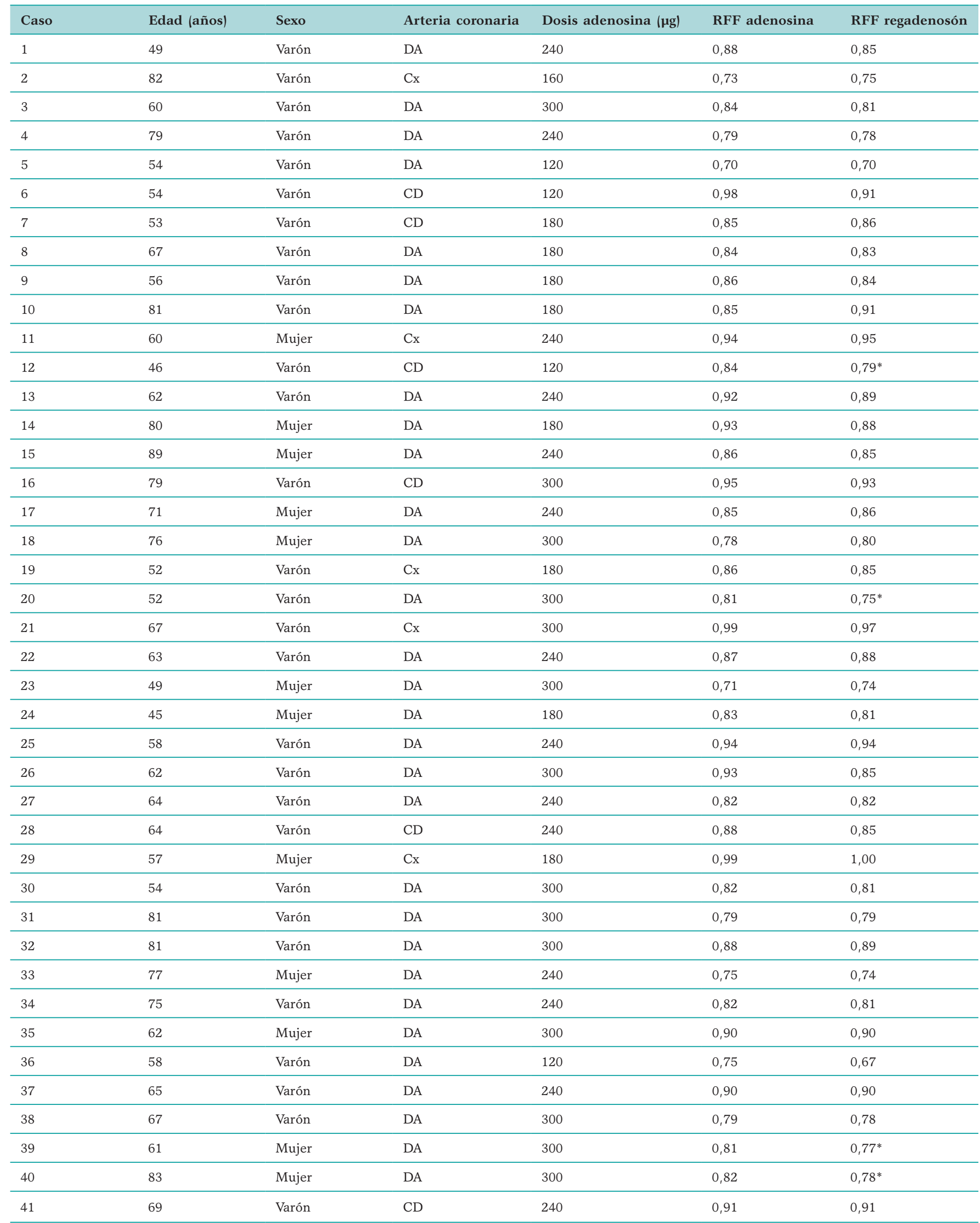

CD: coronaria derecha; Cx: circunfleja; DA: descendente anterior; RFF: reserva fraccional de flujo.

${ }^{*}$ Casos con discrepancias clínicamente relevantes. 


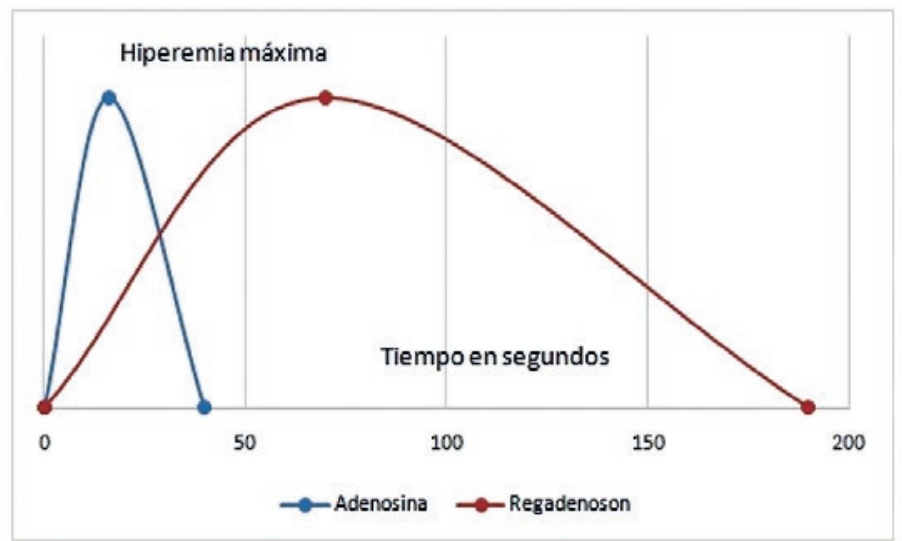

Figura 3. Tiempos de hiperemia. Diferencias en el tiempo hasta alcanzar la hiperemia y su duración (media en segundos) entre la adenosina intracoronaria y el regadenosón intravenoso.

\section{Perfil de los efectos secundarios}

Los efectos secundarios fueron leves con ambos fármacos (tabla 4). Tras la administración de regadenosón, la mayor parte de los pacientes experimentaron algún grado de malestar, que toleraron bien y no impidió proseguir el estudio.

Se observaron 2 casos de bloqueo con pausa mayor de 3 segundos tras la administración de adenosina en la coronaria derecha, que se resolvieron espontáneamente. Con regadenosón no se registraron trastornos de la conducción ni otras complicaciones.

\section{DISCUSIÓN}

La fiabilidad de la medida de la RFF depende de la capacidad para inducir una hiperemia coronaria máxima ${ }^{11}$. El agente farmacológico más utilizado ha sido la adenosina en infusión intravenosa, aunque el uso intracoronario se ha extendido en muchos laboratorios por resultar más rápido y conseguir efectos similares. Las dosis recomendadas son de 100 y $200 \mu \mathrm{g}$ para las coronarias derecha e izquierda, respectivamente. Sin embargo, se sabe que la respuesta a estas dosis difiere en cada paciente. Algunos autores recomiendan dosis de $300 \mu \mathrm{g}$ o incluso mayores, pero estas dosis elevadas provocan con frecuencia efectos adversos, principalmente trastornos de la conducción, que no son deseables en una prueba diagnóstica ${ }^{12}$.

En este trabajo se muestra que el regadenosón en un único bolo intravenoso por vía periférica a dosis fija independientemente del peso y de la función renal podría ser una alternativa a la adenosina. Se ha observado una buena correlación lineal entre la RFF calculada con adenosina intracoronaria o con regadenosón intravenoso $(\mathrm{r}=0,925 ; \mathrm{p}<0,001)$.

Además, en el análisis comparativo se observó que el bolo de regadenosón intravenoso consiguió valores de RFF significativamente menores que los obtenidos con el uso de bolos de adenosina intracoronaria (diferencia de 0,014 $\pm 0,028$; intervalo de confianza del 95\%, 0,005-0,023; $\mathrm{p}=0,002)$.

Probablemente, la mayor hiperemia conseguida con el regadenosón fue el motivo por el que en cuatro casos con RFF $>0,80$ tras la administración de adenosina se obtuvieron valores de $\mathrm{RFF} \leq 0,80$ al administrar regadenosón, lo que supuso reclasificar la lesión como hemodinámicamente significativa.
Tabla 4. Síntomas y episodios adversos durante la medida de la reserva fraccional de flujo

\begin{tabular}{llll}
\hline Evento & Adenosina & Regadenosón & $\mathbf{p}$ \\
\hline Síntomas* & $11(27)$ & $30(73)$ & 0,29 \\
\hline Asistolia $>3$ segundos & $2(5)$ & 0 & $<0,001$ \\
\hline Otras complicaciones & 0 & 0 & - \\
\hline
\end{tabular}

Los datos expresan $\mathrm{n}(\%)$

*Disnea, malestar torácico, cefalea, rubor o náuseas.

Por otra parte, la administración exacta de la dosis de adenosina intracoronaria requería el posicionamiento del catéter guía dentro del ostium coronario, lo que no siempre fue posible y por ello la dosis administrada a veces no fue la establecida.

Así pues, debido a una dosis insuficiente o a una técnica imprecisa el hecho es que en un $9,8 \%$ de los casos no se consiguió una hiperemia máxima con la adenosina. Nuestros datos concuerdan con estudios previos que han puesto de manifiesto que hasta un $10 \%$ de los casos podrían presentar una hiperemia subóptima con adenosina intracoronaria ${ }^{13,14}$. En la misma línea, un metanálisis reciente mostró diferencias en la RFF similares a las observadas en nuestro trabajo cuando se comparaba la administración de adenosina intracoronaria respecto a la infusión intravenosa ${ }^{15}$. Este hecho podría resultar relevante para indicar o no una revascularización.

Otro aspecto interesante del regadenosón es el mantenimiento más prolongado de la hiperemia máxima. Aunque en nuestro trabajo solo se valoraron vasos con lesiones focales, el mayor tiempo medio de hiperemia observado con regadenosón $(174 \pm 72$ s) podría resultar útil para efectuar medidas múltiples de la RFF en vasos con lesiones en serie o enfermedad difusa.

Una potencial limitación del regadenosón es su mayor coste respecto a la adenosina. En este sentido, y a falta de estudios de coste-eficacia, se ha reportado un uso de los recursos más eficiente cuando se administró regadenosón en comparación con adenosina y dipiridamol en la prueba de estrés con isótopos ${ }^{16}$.

Finalmente, en nuestro estudio los efectos adversos fueron muy leves con ambos fármacos. Sin embargo, son bien conocidos los efectos arritmogénicos y dromotrópicos negativos de la adenosina. El regadenosón en bolo único a dosis fija por vía intravenosa periférica mostró un buen perfil de seguridad, y complicaciones 
como el broncoespasmo y el bloqueo auriculoventricular de alto grado parecen ser excepcionales. En raras ocasiones, el efecto vasodilatador más prolongado del regadenosón podría resultar indeseable, en cuyo caso puede administrarse un bolo de $50 \mathrm{mg}$ de aminofilina intravenosa para revertir su efecto ${ }^{17}$.

\section{Limitaciones}

Los datos presentados deberían interpretarse teniendo en cuenta una serie de limitaciones. Se trata de un análisis observacional, abierto, unicéntrico y con una muestra pequeña, por lo que los resultados pueden estar afectados por factores de confusión propios de este tipo de estudios.

A pesar de la corta vida media de la adenosina intracoronaria, no es posible descartar que su administración previa pueda alterar el efecto posterior del regadenosón por fenómenos de precondicionamiento.

La máxima dosis de adenosina utilizada fue a criterio del operador y podría haber resultado insuficiente para inducir la hiperemia máxima.

Por último, no puede obviarse que nuevos índices no hiperémicos han irrumpido con fuerza en el escenario de la fisiología coronaria y se postulan como una alternativa para la valoración funcional de las estenosis sin necesidad de emplear agentes vasodilatadores.

\section{CONCLUSIONES}

La administración de regadenosón en bolo único intravenoso ha mostrado una mayor eficacia en la valoración de la RFF que la administración de bolos de adenosina intracoronaria, y las diferencias observadas podrían resultar relevantes en la toma de decisiones clínicas. Por su facilidad de uso y su buen perfil de seguridad, el regadenosón se presenta como una alternativa útil en la valoración hemodinámica de las estenosis coronarias intermedias.

\section{CONFLICTO DE INTERESES}

Ninguno.

\section{¿QUÉ SE SABE DEL TEMA?}

- La medida de la RFF es una valiosa herramienta en la valoración funcional de estenosis coronarias intermedias.

- Para su cálculo se necesita una hiperemia máxima, y como agente vasodilatador, el regadenosón, un agonista selectivo de los receptores A2a de la adenosina, se presenta como una opción, con la ventaja de que precisa un único bolo intravenoso a dosis fija independientemente del peso y de la función renal del paciente.

\section{¿QUÉ APORTA DE NUEVO?}

- Varios estudios han comparado el regadenosón y la adenosina intravenosa, pero no nos consta ningún trabajo que haya comparado el regadenosón con adenosina intracoronaria.
- En nuestro estudio se ha observado que el bolo de regadenosón intravenoso consigue valores de RFF significativamente menores que los obtenidos con el uso de bolos de adenosina intracoronaria.

- Esta mayor eficacia en la medida de la RFF, fundamental para indicar o no la revascularización, su facilidad de uso y su buena tolerabilidad, hacen que el regadenosón sea una opción a tener en cuenta en la valoración funcional mediante la RFF.

\section{BIBLIOGRAFÍA}

1. Neumann FJ, Sousa-Uva M, Ahlsson A, et al. ESC Scientific Document Group. 2018 ESC/EACTS Guidelines on myocardial revascularization. Eur Heart J. 2019;40:87-165.

2. De Bruyne $\mathrm{B}, \mathrm{Pijl}$ s $\mathrm{NH}$, Barbato $\mathrm{E}$, et al. Intracoronary and intravenous adenosine 5 'triphosphate, adenosine, papaverine, and contrast medium to assess fractional flow reserve in humans. Circulation. 2003;107:1877-1883.

3. Nair PK, Marroquin OC, Mulukutla SR, et al. Clinical utility of regadenoson for assessing fractional flow reserve. J Am Coll Cardiol Intv. 2011;4:10851092 .

4. Arumugham P, Figueredo VM, Patel PB, et al. Comparison of intravenous adenosine and intravenous regadenoson for the measurement of pressure-derived coronary fractional flow reserve. EuroIntervention. 2013;8: 1166-1171.

5. Prasad A, Zareh M, Doherty R, et al. Use of regadenoson for measurement of fractional flow reserve. Catheter Cardiovasc Interv. 2014;83:369-374.

6. VanNunen LX, Lenders GD, Schampaert S, et al. Single bolus intravenous regadenoson injection versus central venous infusion of adenosine for maximum coronary hyperaemia in fractional flow reserve measurement. EuroIntervention. 2015;11:905-913.

7. Stolker JM, Lim MJ, Shavelle DM, et al. Pooled comparison of regadenoson versus adenosine for measuring fractional flow reserve and coronary flow in the catheterization laboratory. Cardiovasc Revasc Med. 2015;16:266-271.

8. Lee JZ, Singh N, Nyotowidjojo I, et al. Comparison of regadenoson and nitroprusside to adenosine for measurement of fractional flow reserve: A systematic review and meta-analysis. Cardiovasc Revasc Med. 2018;2:168174 .

9. Toth GG, Johnson NP, Jeremias A, et al. Standardization of fractional flow reserve measurements. J Am Coll Cardiol. 2016;7:742-753.

10. Adjedj J, Toth GG, Johnson NP, et al. Intracoronary adenosine: doseresponse relationship with hyperemia. J Am Coll Cardiol Intv. 2015;8:14221430.

11. Pijls NH, De Bruyne B, Peels $K$, et al. Measurement of fractional flow reserve to assess the functional severity of coronary artery stenoses. $\mathrm{N} \mathrm{Engl}$ J Med. 1996;334:1703-1708.

12. Lopez-Palop R, Carrillo P, Frutos A, et al. Comparison of effectiveness of high-dose intracoronary adenosine versus intravenous administration on the assessment of fractional flow reserve in patients with coronary heart disease. Am J Cardiol. 2013;111:1277-1283.

13. Jeremias A, Whitbourn RJ, Filardo SD, et al. Adequacy of intracoronary versus intravenous adenosine-induced maximal coronary hyperemia for fractional flow reserve measurements. Am Heart J. 2000;140:651-657.

14. Casella G, Leibig M, Schiele TM, et al. Are high doses of intracoronary adenosine an alternative to standard intravenous adenosine for the assessment of fractional flow reserve? Am Heart J. 2004;148:590-595.

15. Rigattieri S, BiondiZoccai G, Sciahbasi A, et al. Meta-Analysis of Head-toHead Comparison of Intracoronary Versus Intravenous Adenosine for the Assessment of Fractional Flow Reserve. Am J Cardiol. 2017;120:563-568.

16. Friedman M, Spalding J, Kothari S, et al. Myocardial perfusion imaging laboratory efficiency with the use of regadenoson compared to adenosine and dipyridamole. J Med Econ. 2013;16:449-460.

17. Al Jaroudi W, Iskandrian AE. Regadenoson: A New Myocardial Stress Agent. J Am Coll Cardiol. 2009;54:1123-1130. 